\title{
Téoros
}

Revue de recherche en tourisme

\section{Les nouveaux outils du touriste d'affaires}

\section{François Bédard}

Volume 15, numéro 3, automne 1996

Le tourisme d'affaires

URI : https://id.erudit.org/iderudit/1075067ar

DOI : https://doi.org/10.7202/1075067ar

Aller au sommaire du numéro

Éditeur(s)

Université du Québec à Montréal

ISSN

0712-8657 (imprimé)

1923-2705 (numérique)

Découvrir la revue

Citer cet article

Bédard, F. (1996). Les nouveaux outils du touriste d'affaires. Téoros, 15(3),

42-44. https://doi.org/10.7202/1075067ar d'utilisation que vous pouvez consulter en ligne.

https://apropos.erudit.org/fr/usagers/politique-dutilisation/ 


\section{LES NOUVEAUX OUTILS DU TOURISTE D'AFFAIRES}

François Bédard, professeur

Département d'études urbaines et touristiques - UQAM

Liessor sans précédent au cours des dernières années - et le mouvement ne semble pas ralentir - des nowelles technologies de l'information et des télécommunications facilite le travail à l'exterieur da bureau. Par exemple, la miniaturisation des equipements d'informatique et de bureautique ainsi que les appareils de conmunication sans fil permenent aux voyageurs d'affaires de littéralement voyager avec un * bureau mobile s.

Conscients de l'impontance du segment de marché que représente le tourisme d'affaires et desireux de toujours mieux le desservir, les intervenants de l'industrie du tourisme et du woyage, notamment les compagnies de transpon et d'hébergement, les agences de voyages et les organisateurs de congrès, ont intégré a leur gamme de services de nouveaux outils technologiques concus pour augmenter leur propre productivité ainsi que celle de leur clientele et, il est important de le souligner, pour s'assurer de leur fidélité. Dans les lignes qui suivent, nous passons en revue quelques-uns de ces nouveaux outils technologiques du touriste d'affaires par type d'intervenants.

\section{LES COMPAGNIES DE TRANSPORT}

Les compagnies de transport (avion, autocar, train) rivalisent d'ingéniosité pour s'attirer la clientèle d'affaires. En plus d'offrir à leurs clients des sièges plus spacieux et un service à bord hors pair, ils mettent à leur disposition une panoplie de services rendus possibles grâce aux nouvelles technologies.

Pendant leurs déplacements, les gens d'affaires sont tenus bien informés de I"actualitế au moyen de la projection sur vidéo de bulletins de nouvelles produits par des compagnies de télévision offrant des informations en continu.

Plusieurs compagnies de transport offrent à bord un service de téléphone cellulaire permettant à leur clientèle d'effectuer des communications pendant le trajet.

La clientèle d'affaires et de grands voyageurs est également bien servie aux points de départ, de transit ou de destination. Des salons aménagés à leur intention sont munis, entre autres, de systèmes de télécommunications permettant l'envoi ou la réception de données, rendant ainsi plus productifs les temps d'attente.

L'informatisation et l'automatisation de la gestion ont rendu les procédures de réservation directe plus simples et plus rapides. Ce service est très prisé par les voyageurs d'affaires souvent forcés d'effectuer à la dernière minute des réservations ou des modifications à leur itinéraire.
L'informatique a également permis de simplifier d'autres procédures. A titre d'exemple, certaines compagnies aériennes ont éliminé le besoin de se procurer un titre de transports, c'est-à-dire le billet papier (Ticketless travel). Dans ce cas, seul un numero de confirmant la réservation et le paiement suffit pour l'embarquement. Mentionnons toutefois que celte pratique n'est pas encore genéralisée.

La technologie du alobal Positioning System. est utilisée à bord de certains avions pour montrer aux voyageurs la progression du trajet et indiquer, reperes geographiques à l'appui, le circuit parcouru et à parcourir. Cette technologie, constituée d'un réseau de 24 satellites, permet de localiser la progression d'un appareil ou d'un véhicule sur la carte informatisée apparaissant sur un écran cathodique"l". Un petit nombre de compagnies de location d'automobiles ont égatlement commencé à utiliser cette technologie comme système de guidage plus évolué que celui de la carte routière traditionnelle remise à leurs clients au moment de la location. Il suffit d'y entrer une adresse ou un code quelconque et, par effet de zooms successifs, l'ordinateur permet de sélectionner le meilleur trajet et mểme de signaler les erreurs de parcours. Un tel outil de guidage répond aux besoins de l'automobiliste d'affaires desireux de se rendre sans tracas et dans les meilleurs délais au lieu de réunions.

\section{LES COMPAGNIES HÔTELIĖRES}

Les hôtels, particulièrement les grandes chaînes, utilisent fortement les nouvelles 


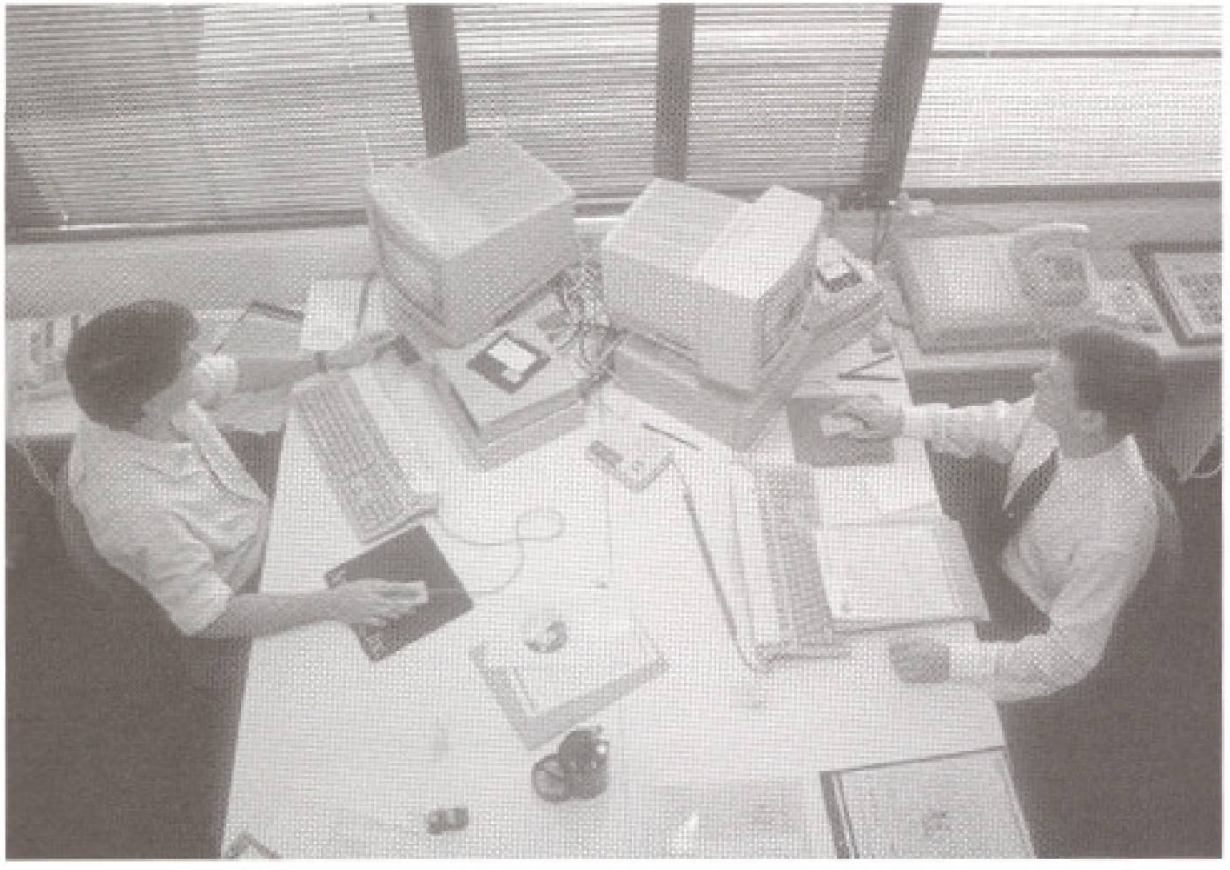

technologies pour mieux servir leurs clienteles d'affaires.

Les technologies ont d'abord été utiliseses pour accélérer les formalitếs à l'arrivée et au départ, faisant ainsi économiser un temps précieux aux voyageurs $\mathrm{d}^{+}$affaires souvent contraints à un horaire serré.

Les hôtels surtout orientés vers la clientèle d'affaires mettent à la disposition de leurs clients une série d'outils technologiques facilitant leur travail pendant un scjour chez eux. Les clients ont accès a un centre d'affaires où ils retrouvent du personnel de secrétariat et de l'équipement d'informatique et de bureautique. Certaines chambres sont dotées d'équipements tels un ordinateur portatif avec modem et un lien Intemet, un télécopieur, une boite vocale, etc. L'appareil de télévision est également de plus en plus utilisé pour des fins utilitaires ou de travail.

Prenons l'exemple de la chaine Delta. Lors du congrès 95 d'ACTA-Québec portant sur les nouvelles technologies, un de ses représentants de cette chaine indiquait que le fait de se procurer et de développer les outils technologiques de pointe permet de se munir d'un atout compétitif. Les hôtels Delta utilisent ces technologies dans trois buts principaux :

1) Améliorer l'expérience du client. Cela se traduit, par exemple, par la création chez Delta de la aZone Affairess pour mieux répondre aux besoins particuliers de la clientèle d'affaires. II s"agit de lui foumir des endroits entièrement équipés de micro-ordinateurs, de télécopieurs, d'équipement de vidéod'améliorer I'expérience du client dès le tout début en créant un site Internet facile d'utilisation et attrayant. La compagnie enregistre d'ailleurs environ 2500 consultations (frappes) par jour dans son site Internet.

2) Améliorer l'efficacité opérationnelle, pour consacrer plus de temps à la satisfaction de la clientèle. Chec Delta, on mise beaucoup sur les outils technologiques pour les domaines de la collecte et la gestion de donnces, le contrôle de la qualité, les finances et la gestion de temps, entre autres. Le temps, l'argent et l'énergie économisés peuvent ainsi être investis dans des actions visant à satisfaire la clientèle.

3) Pousser l'eftort de marketing. Cela se traduit, entre autres, par la mise sur pied d'un programme de fidélisation ainsi que d'une vaste banque de données se voulant commune et accessible à tous les hôtels. Delta, peu importe leur situation géographique dans le monde. C'est done l'amélioration des systèmes de réseaux qui est l'un des conférence, etc. II s'agit également enjeux les plus importants. On travaille fort à l'interconnectivité des bases de données.

\section{LES AGENCES DE VOYAGES}

Les agences de voyages spécialisées dans la clientèle d'affaires connaissent les grandes exigences de cette clientềle et doivent être bien outillées pour la satisfaire. Elles possèdent les technologies requises (ex. systèmes de réservation informatisés) et savent comment les utiliser de façon optimale pour rechercher des informations pointues; certaines agences foumissent même à leurs clients les outils nécessaires pour y accéder, comme une banque d'information multimedia sur Internet ou sur un CD-ROM ${ }^{(5)}$.

Pour illustrer la réalité des agences de voyages spécialisées dans le tourisme d'affaires, prenons l'exemple d'American Express et de Carlson Wagonlit.

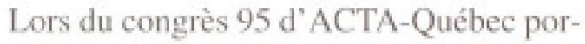
tant sur les nouvelles technologies, une représentante d'American Express Canada ${ }^{\text {al }}$ indiquait que les priorités de sa compagnie en matière d'orientations technologiques consistaient en l'amélioration de la performance du système informatisé de réservations et le développement de nouveaux outils permettant de contrôler la qualité. Un des outils qui sera privilégié dans ces domaines est la Carte intelligente (Smart Card), c'est-à-dire une carte à puce qui sera presqu un système informatique en soi. Des outils utilisant un système de reconnaissance de la parole (Voice Recognition System) seront égulement utilisés. Bien entendu, on développera l'Internet et des CD-ROM. Cependant, nul ne sait le temps nécessaire pour que ces technologies ne soient vraiment intégrées aux opérations cou= rantes d'American Express Canada, mais tout indique que cela se fera dans un avenir assez rapproché.

Des outils tels qu' Internet et les Systèmes de Réservation Informatisés sont des êlements très importants de l'accroissement du réseau de distribution des produits touristiques. En effet, selon le représentant de la compagnie Carlson Wagonlit ${ }^{\text {(5) }}$ participant au congrès 95 d'ACTAQuébec, on constate dans le monde du voyage un mouvement de réservation sans intermédiaire (Agentless reserva- 
tion). Nous commençons d'ores et déjà à affronter cette dure réalité. A ce jour, $2 \%$ des réservations de l'industrie touristique se font via Internet. Mais on prévoit que d'ici l'an 2004, cest-â-dire dans moins de dix ans, $7 \%$ des réservations se feront par l'entremise de ce réseau. Pour une industrie avec un chiffre d'affaires de 9,5 milliards de dollars, ce $5 \%$ d'augmentation représente une perte de cinq cent millions de dollars. L'industrie du voyage subira probablement des changements importants d'ici quelques années.

\section{LES ORGANISATEURS DE CONGRÈS}

L'industrie des congrès et des réunions représente un important segment du tourisme d'affaires. A titre d'exemple de nouvel outil dans ce secteur, mentionnons *Avantages Montréals. Récemment des intervenants de l'industrie des congrès ât Montréal, sous la coordination de Events: Intemational et avec la participation de la Chaire de Tourisme de I'UQAM, ont forme un regroupement pour lancer un nouvel outil de marketing connu sous le

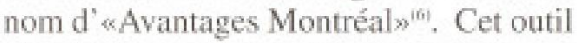
vise a donner des avantages concurrenciels significatifs à Montreal comme ville de congrès.

Avantages Montréal comporte deux volets: Crédit Montréal, un programme de financement provisoire et Privilèges Montréal, un large éventail de bonifications financières.

Crédit Montréal consiste en une marge de crédit consentie par une institution bancaire, à certaines conditions, à toute association tenant un congrès d denvergure à Montréal. Pendant les années qui précèdent le congrès, l'équipe mandatée pour organiser l'événement pourra alors consacrer tous ses efforts à la préparation plutôt qu'à la recherche de financement. Lassociation aura accès a cette marge de crédit jusqu' au moment de ses entréses de fonds.

Grâce au volet Privilèges Montréal, l'association dispose gratuitement d'avantages particulièrement appréciables dont la valeur peut atteindre $250000 \$$. Entre autres privilèges, mentionnons: l'aménagement du site (décoration du hall et signalisation); le service de courtage en

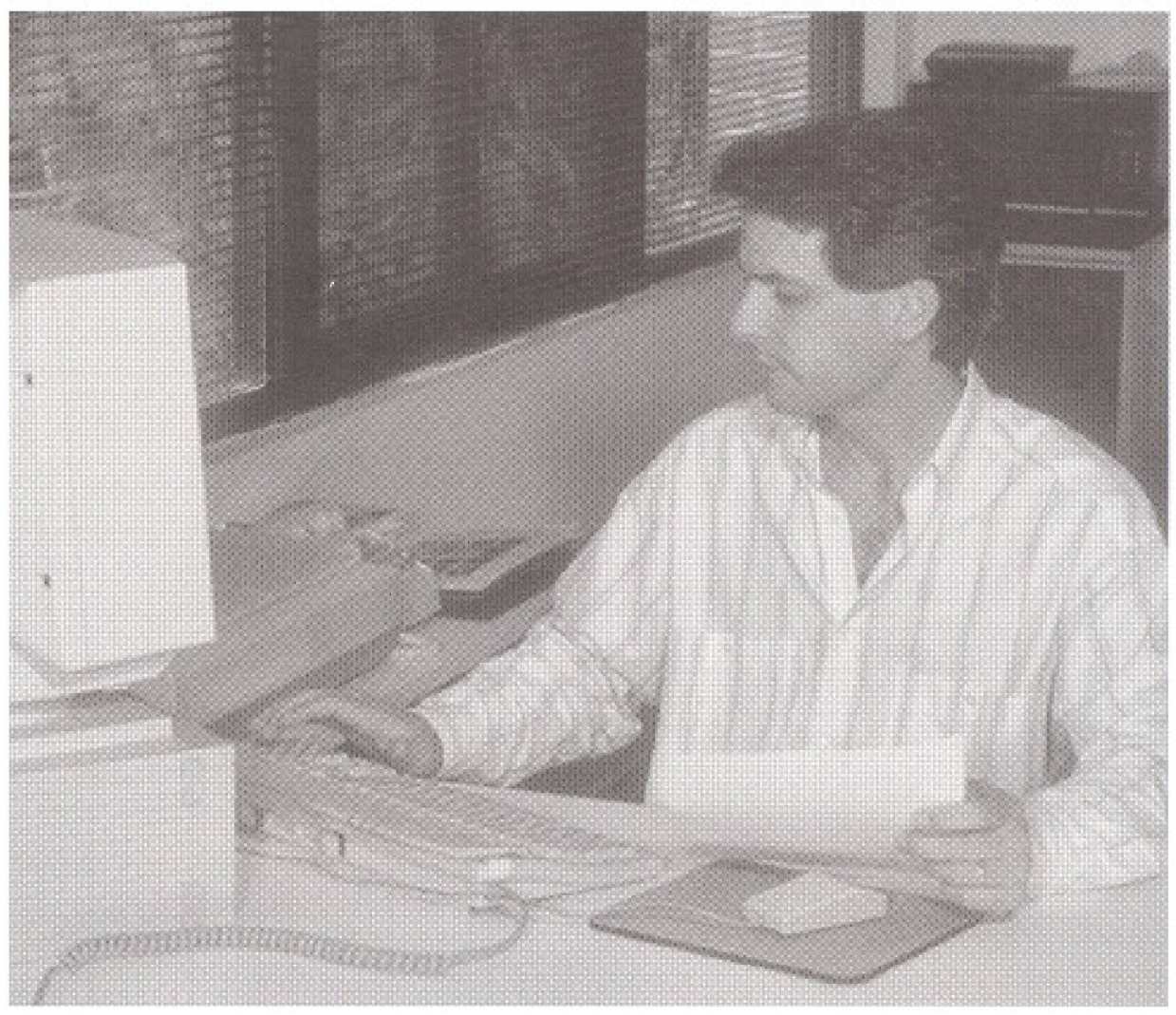

douanes; le service de messagerie vocale à la disposition des congressistes; le système de vote êlectronique; quatre billets d'avion (en Amérique du Nord); le service de navette; hôtesses, guides et personnel de soutien; design graphique, documents de soumission, diaporama, soutien promotionnel; accès au réseau international d'Avantages Montréal pour la promotion de son événement à travers le monde; et l'utilisation des installations du théâtre d'avant-garde de la métropole (groupes de $3000,5000,10000$ et 15000 personnes).

\section{CONCLUSION}

Comme nous venons de le voir, le touriste d'affaires a accès aujourd' hui à une panoplie de nouveaux outils conçus pour répondre à ses besoins particuliers. Plusieurs imnovations chez les compagnies de transport, les sociétés hôtelières, les agences de voyages et les organisateurs de congrès ont été implantées grâce àt l'evolution des nouvelles technologies de l'information et des télécommunications. Certaines innowations visent à rendre plus productif le travail du touriste d'affaires lors de ses déplacements en mettant à sa disposition ni plus ni moins qu'un bureau mobile. D'autres visent à faire économiser du temps au touriste d'affaires lors des procédures de réservation, d'enregistrement et de paiement ou dans sa recherche d'information voyages. Enfin, d'autres lui conferent des bonifications pécuniaires ou en biens et service fort appréciables.

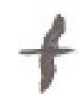

\section{NOTES}

(1) CAZELAIS, Nomand (1994), Cartes à la rescousse, Le Devoir, Tourisme d'affaires Chronique 3-8, Montréal, 21 teverier.

(2) Actes du congrès $95 \mathrm{~d}^{2}$ ACTA-Québec, Chaire de Tourisme de l'UQAM, Montreal, p. 24-26.

(3) BÉDARD. François et LE ROUX. Philippe (1995), wes agences de voyage vont-elles finir dans le fossé des inforoutes ? $\%$, Teoros, vol. 14, no 3, UQAM, Montréal, p. 8-10.

(4) Les grandes orientations technologiques des chawes, Actes du congres 95 d'ACTAQuébec, UQAM, Montréal, p. 24-26.

(5) Hid

(6) BÉDARD, François (1996), Avaniages Montréal, Chaire de Tourisme de I'UQAM, Montréal. 